\title{
Efecto de la velocidad de rotación y la indentación en soldadura de punto por fricción agitación de aleaciones de aluminio disimilares
}

\author{
Joaquín M. Piccini ${ }^{\mathrm{a}, \bigotimes}$, Hernán G. Svoboda ${ }^{\mathrm{a}, \mathrm{b}}$ \\ ${ }^{a}$ Universidad de Buenos Aires, Facultad de Ingeniería, Laboratorio de Materiales y Estructuras, \\ INTECIN, Grupo de Tecnología de la Soldadura, Ciudad de Buenos Aires, Argentina \\ ${ }^{\mathrm{b}}$ Consejo Nacional de Investigaciones Científicas y Técnicas, Ciudad de Buenos Aires, Argentina \\ ( $\triangle$ Autor para la correspondencia joaquin.piccini@gmail.com)
}

Enviado: 22 Febrero 2016; Aceptado: 20 Febrero 2017; Publicado on-Line: 22 Marzo 2017

\begin{abstract}
RESUMEN: En los últimos años, dentro de a la industria automotriz, se busca introducir partes de aluminio en reemplazo de componentes de acero para la disminución en peso de los vehículos. Estas piezas deben ser unidas entre sí, por ejemplo, mediante soldadura. El proceso de soldadura más utilizado dentro de la industria automotriz es la soldadura de punto por resistencia (RSW). Sin embargo, la soldadura RSW de aleaciones de aluminio presenta desventajas importantes. Dentro de este contexto se ha desarrollado una variante del proceso de soldadura por Fricción Agitación denominado Soldadura de Punto por Fricción Agitación (FSSW), el cual presenta un fuerte interés en relación a la soldadura de aleaciones de aluminio y de materiales disímiles en chapas finas. Los parámetros de soldadura afectan a las características de las juntas obtenidas. Sin embargo, es escasa la información disponible sobre la influencia de estos sobre las características de la unión, en particular para juntas disimilares y en chapa fina. El objetivo de este trabajo fue estudiar el efecto de la velocidad de rotación y la profundidad de indentación de la herramienta sobre las características de uniones disimilares soldadas por FSSW. Uniones soldadas sin defectos pudieron obtenerse con propiedades mecánicas superiores a lo que se reporta hasta el momento. La carga de rotura máxima fue de $5800 \mathrm{~N}$. La longitud efectiva de unión aumentó con la indentación de la herramienta, mientras que la carga de rotura aumenta y luego disminuye. Finalmente, al soldar con 1200 RPM las propiedades mecánicas de las uniones disminuyeron, respecto a lo obtenido con 680 y 903 RPM.
\end{abstract}

PALABRAS CLAVE: Aleaciones de aluminio; Peel test; Soldadura de punto por fricción; Uniones disimilares

Citar como / Citation: Piccini, J.M.; Svoboda, H.G. (2017) "Efecto de la velocidad de rotación y la indentación en soldadura de punto por fricción agitación de aleaciones de aluminio disimilares". Rev. Metal. 53(1):e090. http://dx.doi. org/10.3989/revmetalm.090

ABSTRACT. Effect of tool rotational speed and penetration depth on dissimilar aluminum alloys friction stir spot
welds. In the last years, the automotive industry is looking for the use of aluminum parts in replace of steel parts
in order to reduce the vehicles weight. These parts have to be joined, for instance, by welding processes. The
more common welding process in the automotive industry is the Resistance Spot Welding (RSW) technique.
However, RSW of aluminum alloys has many disadvantages. Regarding this situation, a variant of the Friction
Stir Welding process called Friction Stir Spot Welding (FSSW) has been developed, showing a strong impact in
welding of aluminum alloys and dissimilar materials in thin sheets. Process parameters affect the characteristics
of the welded joints. However, the information available on this topic is scarce, particularly for dissimilar joints 
and thin sheets. The aim of this work was to study the effect of the rotational speed and the tool penetration depth on the characteristics of dissimilar FSS welded joints. Defects free joints have been achieved with higher mechanical properties than the ones reported. The maximum fracture load was $5800 \mathrm{~N}$. It was observed that the effective joint length of the welded spots increased with the tool penetration depth, meanwhile the fracture load increased and then decreased. Finally, welding at 1200 RPM produced welded joints with lower mechanical properties than the ones achieved at 680 and 903 RPM.

KEYWORDS: Aluminum alloys; Dissimilar welding; Friction Stir Spot Welding; Peel test

ORCID ID: Joaquín M. Piccini (http://orcid.org/0000-0001-7968-3382); Hernán G. Svoboda (http://orcid. org/0000-0001-7539-8306)

Copyright: ( 2017 CSIC. Este es un artículo de acceso abierto distribuido bajo los términos de la licencia Creative Commons Attribution (CC BY) España 3.0.

\section{INTRODUCCIÓN}

Existe un marcado interés en la industria del transporte por aumentar la eficiencia en el uso de combustibles y así disminuir la emisión de contaminantes al medio ambiente. Para ello, entre otras cosas, se busca reemplazar en la fabricación de vehículos, componentes de aleaciones ferrosas por aleaciones livianas de aluminio o magnesio, con un comportamiento mecánico similar, a fin de disminuir el peso de los mismos (Mishra y Mahoney, 2007; Wang y Lee, 2007). En este contexto, toma un papel preponderante el estudio y desarrollo de distintas tecnologías de unión para aplicarse sobre los diversos componentes fabricados con dichas aleaciones.

Uno de los procesos más difundidos, de especial importancia en la industria automotriz, es la Soldadura de Punto por Resistencia (Resistance Spot Welding, RSW) (Shome y Tumuluru, 2015). Sin embargo, la soldadura por resistencia de aleaciones de aluminio presenta ciertas limitaciones. En primer lugar, la vida del electrodo disminuye significativamente en relación a la soldadura de acero, la cantidad de energía involucrada es muy elevada y la generación de defectos (porosidad) en la región de la unión se ve incrementada, por lo que las propiedades mecánicas de la unión soldada son menores que las de los materiales base (European Aluminium Association, 2015). En respuesta a éstos problemas, en el año 2003 Mazda Motor Corporation desarrolló un nuevo proceso de soldadura en estado sólido denominado Soldadura de Punto por Fricción Agitación (FSSW, según lo indican las siglas en inglés). Este proceso surgió como una variante de aquel creado en el Reino Unido en el año 1991 por The Welding Institute, denominado Soldadura por Fricción Agitación (FSW) (Mishra y Mahoney, 2007).

La Soldadura de Punto por Fricción Agitación consiste en una herramienta rotante, con una geometría particular, que se inserta en una junta solapada, permaneciendo un dado período de tiempo y siendo finalmente extraída. Durante la inserción de la herramienta, el hombro de la misma genera el calentamiento del material por fricción, mientras que el pin produce la agitación del material plastificado, produciendo la soldadura en fase sólida de ambas chapas.

FSSW presenta numerosas ventajas que han posicionado a este proceso como una alternativa al proceso RSW, sobre todo en la soldadura de aleaciones de difícil soldabilidad por métodos tradicionales de fusión. Por tratarse de una soldadura en estado sólido, la energía que se le entrega al material en forma de calor es menor que la correspondiente a cualquier proceso de soldadura por fusión. Por lo tanto, el tamaño de la zona afectada por el calor (HAZ, de acuerdo a sus siglas en inglés) es menor, así como las tensiones residuales y las distorsiones inducidas en el material producto de la soldadura. De igual manera, al disminuir el aporte térmico sobre las chapas, se reduce en cierto grado la degradación de las propiedades mecánicas que han sido obtenidas previamente por tratamientos térmicos o termomecánicos (Ferjutz y Davis, 1993; Jenney y O’Brien, 2001; Kou, 2003).

Los parámetros del proceso presentan un efecto significativo en la conformación del punto de soldadura por FSSW. Entre ellos, la geometría de la herramienta, la velocidad de rotación de la herramienta, el tiempo de permanencia, la profundidad de la penetración de la herramienta, la preparación superficial de la junta y los tratamientos térmicos post-soldadura son algunos de los más significativos (Tozaki et al., 2007; Tran et al., 2009; Yuan et al., 2011; Zhang et al., 2011; Francesco y Svoboda, 2013).

La resistencia de las uniones soldadas es un aspecto crítico cuando la soldadura de punto por fricción agitación es utilizada sobre componentes estructurales de automóviles. Dicha resistencia se ve estrictamente relacionada con los parámetros del proceso y la geometría de la herramienta. Es reportado en la bibliografía como el diámetro del hombro y su forma, la forma del pin, su longitud y diámetro afectan a la generación de calor y al flujo de material plastificado (Lathabai et al., 2006; Tozaki et al., 2007; Tran et al., 2009; Rao et al., 2015). 
Uniones soldadas libres de defectos y con una buena conformación del punto presentarán una alta carga de arrancamiento en ensayos del tipo Peel Test (PT), con un modo de fractura circunferencial. Por el contrario, puntos de soldadura de bajo rendimiento fallarán de un modo interfacial, asociado a menores cargas de rotura (Yuan et al., 2011; Francesco y Svoboda, 2013).

En relación a los parámetros de soldadura, diversos autores han reportado que la carga de rotura en uniones soldadas mediante FSSW, primero se ve incrementada con el aumento de la velocidad de rotación de la herramienta, pero para velocidades muy elevadas, la carga de rotura disminuye (Yuan et al., 2011). En este mismo sentido, se ha reportado que el aumento de la resistencia de las uniones se obtiene cuando el tamaño de la zona agitada es maximizado, lo cual a su vez se obtiene al soldar con menores velocidades de rotación (Rao et al., 2015). Sin embargo, otros autores aseguran que se debe soldar con elevadas velocidades de rotación, como por ejemplo 3000 RPM, para optimizar la carga de rotura de las uniones soldadas de Aluminio 6060-T5 (Lathabai et al., 2006).

La literatura disponible hasta el momento muestra diversos resultados en cuanto a la evolución de las propiedades mecánicas de las uniones al variar los principales parámetros del proceso FSSW. Por lo tanto, solo una comparación cualitativa puede hacerse teniendo en cuenta las diferentes aleaciones soldadas, sus diferentes espesores, la geometría de la herramienta, el dispositivo de sujeción y la máquina utilizada para la soldadura. Por otro lado, la utilización de aleaciones de aluminio dentro de la industria automotriz, implica la necesidad de producir uniones soldadas libres de defectos, con propiedades mecánicas similares a las obtenidas en uniones de acero mediante RSW y en chapa fina (Pereira et al., 2010). Sin embargo, producción sistemática de uniones soldadas de aleaciones de aluminio en chapa fina, con propiedades mecánicas elevadas, no se encuentra del todo desarrollada (Pereira et al., 2010). En este sentido, la información disponible acerca de la unión de aleaciones de aluminio disimilares en chapa fina es escasa.

A lo largo de la última década, se ha trabajado en el estudio de la soldadura FSW, tratando de explicar la relación entre los parámetros del proceso y la microestructura resultante en aleaciones de aluminio. Sin embargo, es escasa la información sistemática disponible respecto de la soldadura FSSW de materiales disímiles y el efecto de los parámetros de soldadura sobre las propiedades mecánicas resultantes, sobre todo en chapa fina debido a las complejidades que esto conlleva. El objetivo de este trabajo fue estudiar la soldabilidad de aleaciones de aluminio disimilares AA5052-H32 y AA6063-T6 en chapa fina de 1 y $2 \mathrm{~mm}$ respectivamente, mediante FSSW. En el mismo sentido, se estudió el efecto de la velocidad de rotación y la profundidad de indentación de la herramienta sobre las características de las uniones, para la optimización de las propiedades mecánicas resultantes.

\section{MATERIALES Y MÉTODOS}

\subsection{Materiales base y soldaduras FSSW}

A fin de alcanzar los objetivos propuestos, se realizaron soldaduras FSSW en juntas de solape de aleaciones de aluminio AA5052-H32 y AA6063-T6, con espesores de $1 \mathrm{~mm}$ y $2 \mathrm{~mm}$, respectivamente. Se evaluaron tres velocidades de rotación de la herramienta (680, 903 y 1200 RPM) y diferentes profundidades de indentación de la misma $(\mathrm{H})$ de 0,05 a $0,60 \mathrm{~mm}$. Cabe destacarse que para indentaciones menores a $0,05 \mathrm{~mm}$, no se produce la unión metalúrgica de los materiales en estudio. Por el contrario, para indentaciones mayores a $0,60 \mathrm{~mm}$ se produce una deformación excesiva de la chapa superior, lo que implica un deterioro de la junta, al menos desde un punto de vista superficial.

La disposición de la junta fue con la chapa de AA5052 en la parte superior de la misma en todos los casos de estudio. En la Tabla 1 se muestran las composiciones químicas de las aleaciones utilizadas. Cómo puede verse, el AA6063 corresponde a una aleación del tipo Al-Mg-Si con presencia de $\mathrm{Cu}$, Fe y Zn, termotratable, empleada en condición T6. Su microdureza Vickers fue de 90 HV. El AA5052 corresponde a una aleación Al-Mg, con presencia de Fe. La condición del material es H32 lo que implica un grado de deformación en frío. La microdureza Vickers de este material fue de $70 \mathrm{HV}$.

La herramienta utilizada para la soldadura por FSSW fue construida en un acero para herramientas tipo H13 con un hombro cóncavo de $12 \mathrm{~mm}$ de diámetro. El pin de la herramienta tiene un perfil cónico liso, de $1,3 \mathrm{~mm}$ de largo y $3,5 \mathrm{~mm}$ de diámetro en el extremo. Todos los puntos soldados mediante FSSW fueron realizados con una velocidad de penetración de $19 \mathrm{~mm} \cdot \mathrm{min}^{-1}$ y un tiempo de permanencia de $1 \mathrm{~s}$. Para cada condición de soldadura se realizaron dos tipos de probetas. Una de ellas se utilizó para la caracterización dimensional y

TABla 1. Composición química de las aleaciones utilizadas (\% peso)

\begin{tabular}{cccccccccc}
\hline Aleación & $\mathbf{S i}$ & $\mathbf{F e}$ & $\mathbf{C u}$ & $\mathbf{M n}$ & $\mathbf{M g}$ & $\mathbf{Z n}$ & $\mathbf{N i}$ & $\mathbf{C r}$ & $\mathbf{P b}$ \\
\hline AA6063 & 0,41 & 0,85 & 0,81 & 0,11 & 0,41 & 0,52 & 0,02 & 0,02 & 0,03 \\
AA5052 & 0,06 & 0,25 & 0,01 & 0,01 & 2,38 & 0,005 & - & 0,16 & - \\
\hline
\end{tabular}


macroestructural del punto soldado, así como para la realización del perfil de microdureza Vickers. Por otro lado, el segundo tipo de probeta se utilizó para caracterizar mecánicamente la unión por medio de ensayos de arrancamiento tipo Peel Test (PT).

En la Tabla 2 se aprecia la matriz de ensayos realizada para los distintos parámetros de soldadura utilizados, así como la identificación empleada para cada probeta soldada.

\subsection{Caracterización macro y microestructural}

Los especímenes para el análisis metalográfico fueron seccionados, montados en frío y preparados para observación metalográfica. Las probetas

TABla 2. Parámetros de soldadura estudiados

\begin{tabular}{lcc}
\hline Probeta & Velocidad de Rotación (RPM) & Indentación (mm) \\
\hline $1-680$ & 680 & 0,1 \\
$2-680$ & 680 & 0,2 \\
$3-680$ & 680 & 0,3 \\
$4-680$ & 680 & 0,4 \\
$5-680$ & 680 & 0,5 \\
$6-680$ & 680 & 0,6 \\
$1-903$ & 903 & 0,1 \\
$2-903$ & 903 & 0,2 \\
$3-903$ & 903 & 0,3 \\
$4-903$ & 903 & 0,4 \\
$5-903$ & 903 & 0,5 \\
$6-903$ & 903 & 0,6 \\
$1-1200$ & 1200 & 0,1 \\
$2-1200$ & 1200 & 0,2 \\
$3-1200$ & 1200 & 0,3 \\
$4-1200$ & 1200 & 0,4 \\
$5-1200$ & 1200 & 0,5 \\
$6-1200$ & 1200 & 0,6 \\
\hline
\end{tabular}

fueron pulidas y finalmente atacadas utilizando un reactivo tipo Keller para poder revelar su microestructura (Vander Voort, 2004).

Sobre las probetas preparadas metalográficamente para observación macrográfica se analizó la macroestructura, la existencia de defectos y otros aspectos macrográficos, como el flujo plástico de ambos materiales, empleando una lupa estereoscópica.

Asimismo, se realizó un análisis dimensional de modo de determinar las características principales de los puntos de soldadura. En la Fig. 1 se aprecian las dimensiones relevadas sobre cada punto. El diámetro del hombro de la herramienta se denominó como Dh. La posición vertical del hook (punto donde comienza la unión metalúrgica) se define como la distancia entre dicho hook y la superficie de la chapa superior $(\mathrm{Kr})$. La longitud de unión efectiva (A) es la distancia horizontal comprendida entre la superficie libre del orificio dejado por el pin y el hook. La indentación de la herramienta $(\mathrm{H})$, es la distancia entre la superficie de la chapa superior y el punto más bajo alcanzado por el hombro de la herramienta. El espesor remanente de la chapa inferior debido a la acción del pin se denominó P. Finalmente, la distancia entre el punto inferior del hombro y la interface de las chapas es IH.

Por último, a fin de analizar en mayor detalle el flujo plástico de ambos materiales alrededor de la herramienta y su interacción, se analizó la microestructura de dicha zona a través de la observación mediante microscopía óptica.

\subsection{Propiedades mecánicas}

Las propiedades mecánicas de los puntos de soldadura se determinaron mediante la realización de perfiles de microdureza Vickers y ensayos tipo PT. Las mediciones de microdureza fueron realizadas a la altura de la posición del hook con una carga de $0,3 \mathrm{Kg}$ y un tiempo de permanencia de $10 \mathrm{~s}$, de acuerdo a lo establecido en la norma ASTM E384 (2009), empleando un equipo EMCOTEST

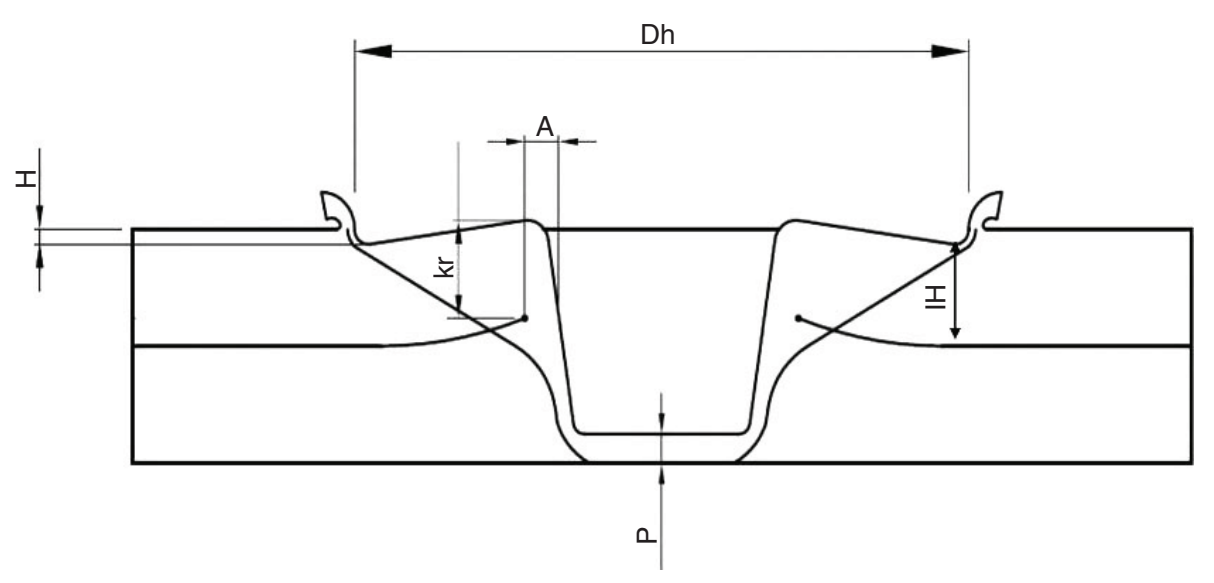

FIgURE 1. Sección transversal de un punto de soldadura y dimensiones relevadas. 
(a)

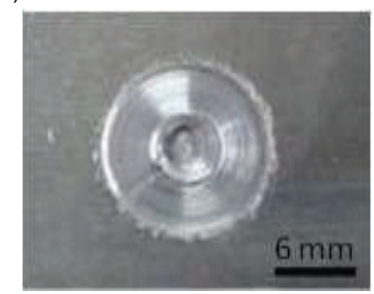

(d)

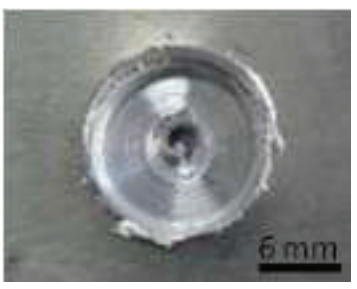

(g)

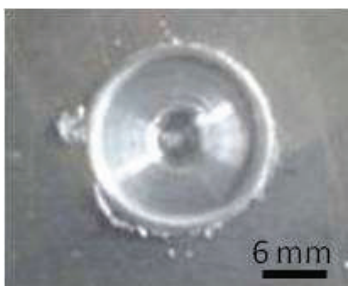

(j)

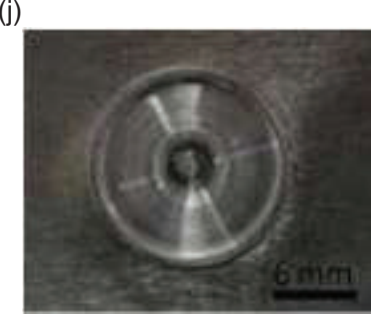

(b)

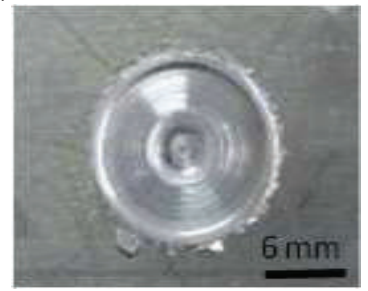

(e)

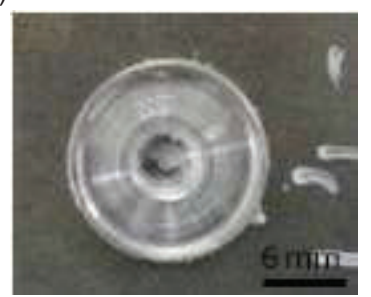

(h)

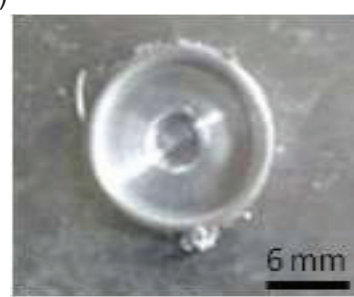

$(\mathrm{k})$

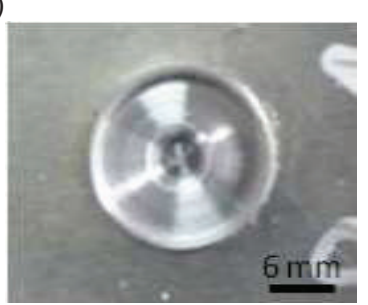

(c)

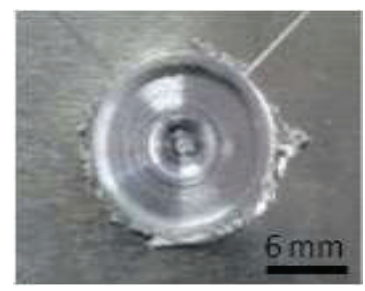

(f)

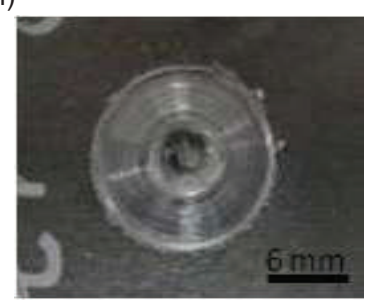

(i)

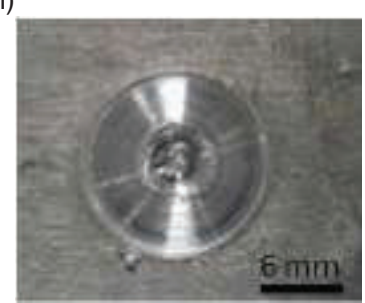

(I)

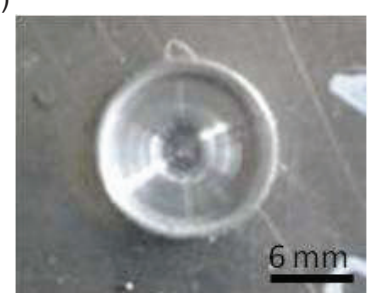

FIGURE 2. Aspecto superficial de los puntos de soldadura: a) 1-680, b) 2-680, c) 4-680, d) 6-680, e) 1-903, f) 2-903, g) 4-903, h) 6-903, i) $1-200$, j) 2-1200, k) 4-1200 y l) 6-1200.

Durascan 10. Los ensayos de PT fueron realizados en una maquina universal Instron TT10-DM con una velocidad de desplazamiento constante de $1 \mathrm{~mm} / \mathrm{min}$. Se ensayaron a rotura tres especímenes de cada condición de soldadura analizada, de modo de tener presente la incertidumbre asociada al proceso. A partir de las curvas carga-desplazamiento obtenidas para cada caso, se determinó la carga máxima de rotura y se analizó el modo de falla. Las superficies de fractura fueron observadas mediante una lupa estereoscópica de campo profundo.

\section{RESULTADOS Y DISCUSIÓN}

\subsection{Aspecto superficial de los puntos de soldados}

En la Fig. 2 se observa cómo evoluciona la conformación del punto con la indentación de la herramienta y la velocidad de rotación de la misma.
Un excelente aspecto superficial se apreció en todos los puntos de soldadura obtenidos bajo las condiciones estudiadas. La penetración de la herramienta afecta a la generación de calor y el flujo de material. Las probetas 1-680, 1-903 y 1-1200 (Fig. 2 (a, e, i)) tienen la menor indentación, mientras que las probetas 6-680, 6-903 y 6-1200 (Fig. 2d y 21) poseen la mayor indentación. Puede observarse el aumento del material que fluye en los alrededores de la herramienta cuando se incrementa la indentación, para todas las velocidades de rotación estudiadas. Para las mayores indentaciones se produce una deformación excesiva de la chapa superior, como se aprecia en las muestras 6-680, 6-903 y 6-1200 (Fig. 2 $(\mathrm{d}, \mathrm{h}, \mathrm{l}))$. Este aspecto para algunas aplicaciones puede ser considerado un defecto y limitaría el nivel de indentación aplicable.

Si bien no existen diferencias sustanciales en el aspecto superficial de los puntos soldados mediante 
FSSW con diferentes velocidades de rotación de la herramienta, se aprecia que para 1200 RPM el aspecto de la superficie del punto soldado presenta una menor rugosidad que cuando se suelda con 680 RPM. Este hecho estaría asociado a la mayor componente de deslizamiento (sliding), respecto de la de arrastre (sticking), para mayores velocidades de rotación (Mishra y Mahoney, 2007). Esto implica una calidad superior de las probetas soldadas con 1200 RPM en términos del aspecto superficial. Este efecto se vuelve más importante para elevados niveles de penetración de la herramienta como se puede ver al comparar la probeta 6-680 (Fig. 2d) con la 6-1200 (Fig. 21).

Es de hacer notar la diferencia que existe en el aspecto superficial del key-hole (perfil del pin de la herramienta de soldadura) de los puntos de soldadura al variar los parámetros del proceso. Puede apreciarse en la Fig. 2, como la zona de transición entre la superficie del hombro y el key-hole depende de los parámetros del proceso. Para bajas velocidades de rotación, esta zona de transición presenta elevada rugosidad superficial. Este efecto disminuye al soldar con 903 RPM y se elimina al soldar con 1200 RPM, para todos los niveles de indentación analizados. Este efecto se cree que también estaría relacionado con la variación de las componentes de deslizamiento y arrastre. Para bajas velocidades de rotación (680 RPM) la componente de arrastre es predominante (Lohwasser y Chen, 2010). Dado el perfil cóncavo del hombro de la herramienta, cierta cantidad de aluminio permanece adherida al mismo y así la calidad de los puntos soldados con 680 RPM no es la óptima. Esto también está relacionado con el corto tiempo de permanencia que se utiliza en el presente trabajo.

Por lo tanto, desde el punto de vista de calidad superficial de las uniones soldadas, al utilizar una velocidad de rotación de 1200 RPM se obtuvieron los mejores resultados, sin importar el nivel de indentación utilizado.

\subsection{Caracterización macroestructural}

En la Fig. 3, pueden apreciarse las macrografías a lo largo del plano de simetría de las secciones transversales de los puntos de soldadura. Se observan las características de los puntos realizados cuando se modifica la indentación de la herramienta y la velocidad de rotación de la misma, sin observarse defectos para las distintas condiciones de soldadura analizadas.

El perfil de la indentación sobre la junta solapada revela la geometría de la herramienta de soldadura utilizada. Puede verse la concavidad del hombro como así también el perfil cónico que presenta el pin. En todas las macrografías se observa un excelente flujo de material entre ambas chapas, en concordancia con lo que se describe en la bibliografía (Yang et al., 2010). Se puede notar que la indentación del hombro en la junta produce una presión radial sobre la chapa superior, produciendo una ligera deflexión de la misma en las adyacencias del hombro de la herramienta (Tran et al., 2009). Este efecto se incrementa a medida que aumenta la indentación (Fig. 3 (g-h)) y está asociado a lo mencionado anteriormente y observado en la Fig. 2 (d, h, 1).

El calor generado en la interface de la herramienta y las chapas a unir produce la plastificación del material cercano a la misma. Al mismo tiempo, el movimiento del pin de la herramienta produce el flujo del material plastificado en la dirección radial y axial (Lathabai et al., 2006). La mezcla entre ambos materiales en estado plástico y la forja producida por la carga axial aplicada por el hombro de la herramienta, producen la unión metalúrgica en estado sólido (Lathabai et al., 2006; Tran et al., 2009; Yang et al., 2010; Zhang et al., 2011). La sección transversal de un punto de soldadura por FSSW puede dividirse en cuatro regiones desde el orificio o key-hole hasta el material base (BM) pasando por la zona agitada (SZ), la zona termomecánicamente afectada (TMAZ) y la zona afectada por el calor (HAZ) (Mishra y Mahoney, 2007). Durante la penetración del pin rotante, una cierta cantidad de material de la chapa superior fluye y es deformado contra la chapa inferior en las adyacencias del pin. Al mismo tiempo, el material de la chapa inferior, calentado y en estado plástico, fluye hacia arriba y hacia las afueras del key-hole (Yang et al., 2010; Yuan et al., 2011). Este mecanismo puede apreciarse en la Fig. 3j.

Puede verse una gran diferencia en el flujo plástico de los puntos soldados con distintas indentaciones. A medida que la indentación de la herramienta aumenta, se ve incrementada la cantidad de material que es extruido hacia el exterior del punto. De igual manera, aumenta el mezclado en la zona agitada y con ello la posición final del hook. Esto implica que la sección resistente, gobernada por la dimensión $\mathrm{A}$, se ve incrementada al aumentar la indentación de la herramienta $(\mathrm{H})$, debido al mayor efecto termomecánico que la herramienta ejerce sobre la interface de las chapas. Sin embargo, al aumentar $\mathrm{H}$, disminuye $\mathrm{Kr}$ (sección resistente de la junta en la dirección vertical).

En la Fig. 3 puede apreciarse la diferencia que existe en el flujo de material plastificado al modificarse la velocidad de rotación de la herramienta. La cantidad de material de la chapa inferior que es agitado por la acción del pin, aumenta con la velocidad de rotación, en concordancia con lo reportado en la bibliografía (Yang et al., 2010). Sin embargo, el aumento de la velocidad de rotación de la herramienta presenta menores niveles de mezcla entre ambas chapas, como se aprecia en las Fig. 3 (a-1). Al soldar con 1200 RPM se puede observar como la posición final del hook tiende a curvarse hacia 
(a)

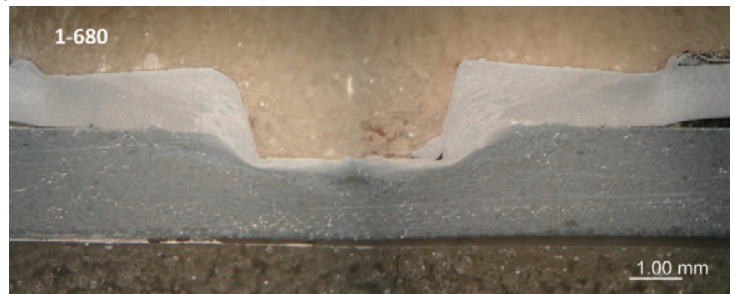

(c)

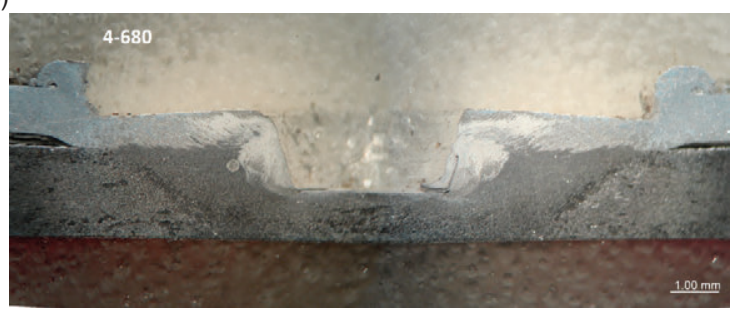

(e)

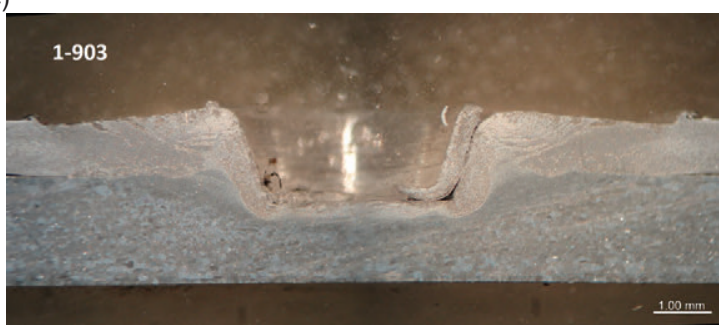

(g)

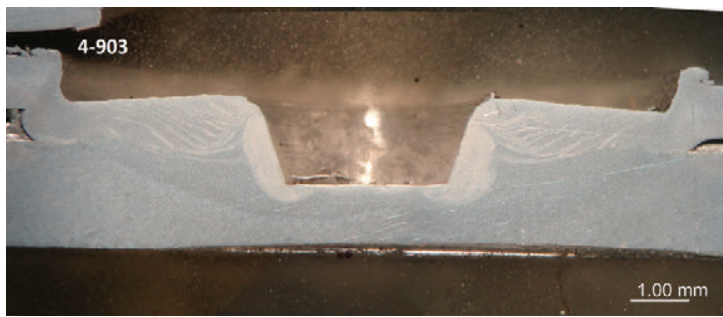

(i)

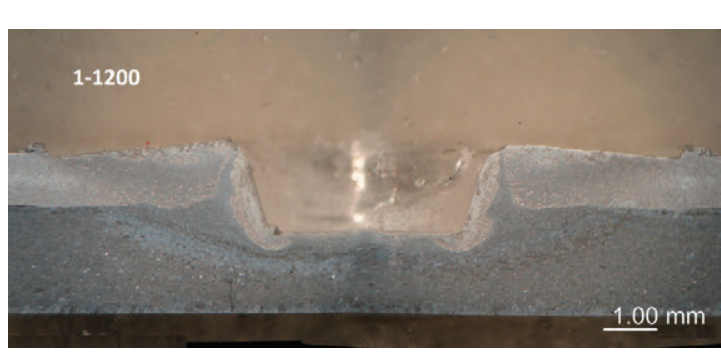

(k)

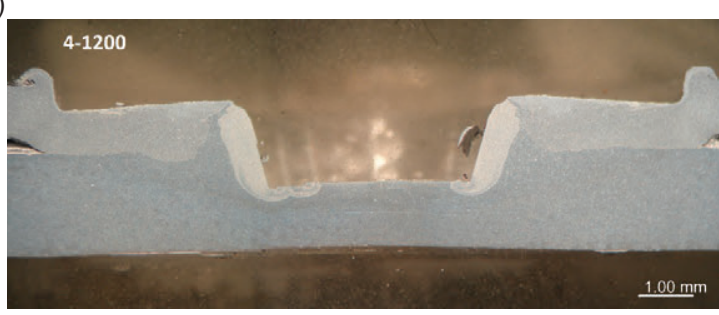

(b)

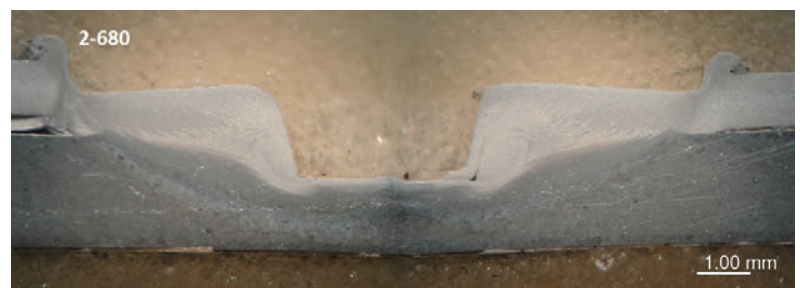

(d)
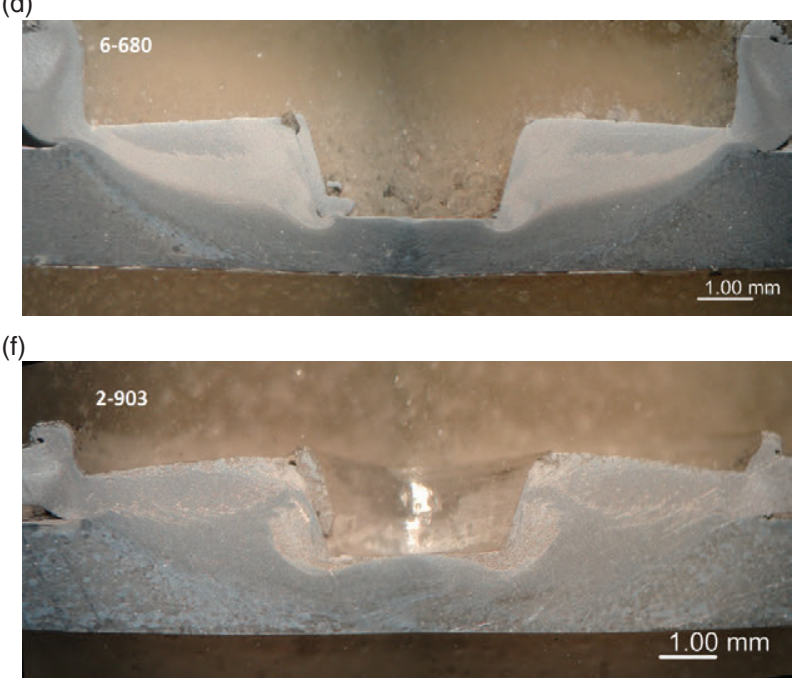

(h)
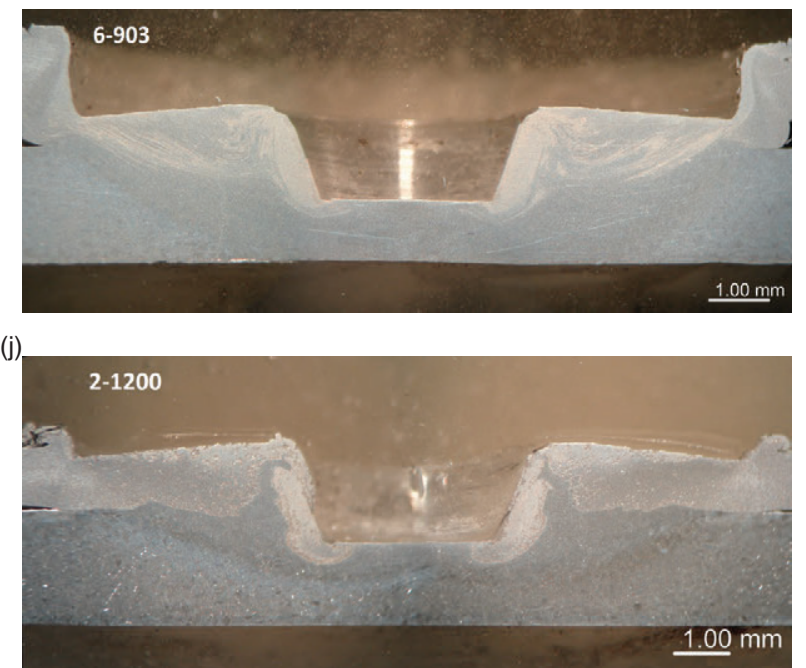

(I)

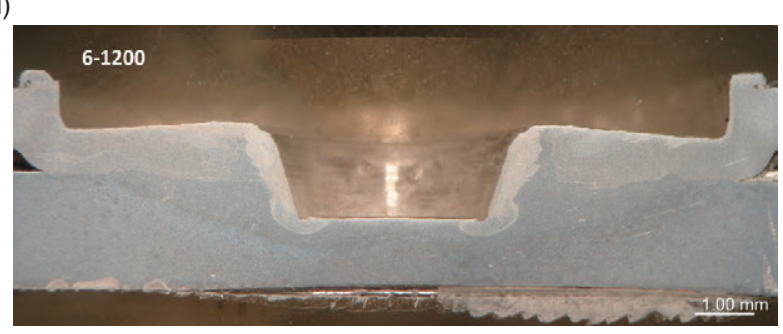

FIGURE 3. Macrografías de las uniones soldadas. 
arriba y hacia zonas más cercanas a la pared lateral del pin. Estos resultados concuerdan con lo previamente reportado en la bibliografía (Yang et al., 2010; Yuan et al., 2011; Rao et al., 2015). Debido a esto, para la soldadura de aleaciones de aluminio en chapa fina, se debe asegurar el correcto flujo plástico utilizando velocidades de rotación de hasta 903 RPM, de acuerdo a los resultados expuestos en el presente trabajo.

\subsection{Análisis dimensional}

Con el fin de caracterizar las uniones soldadas bajo diferentes parámetros de soldadura, se midieron las dimensiones características de cada punto soldado, de acuerdo a lo descripto en la Fig. 1. En la Tabla 3 se aprecian los resultados de dichas mediciones. Las dimensiones características de los puntos de soldadura quedan determinadas por los parámetros operativos empleados y definen la sección resistente del punto (Wang y Lee, 2007; Francesco y Svoboda, 2013). El flujo de material no solo dependerá de la acción de la herramienta, sino también, de las propiedades térmicas y mecánicas de los materiales a unir.

La dimensión IH permite describir que tan cerca estuvo la herramienta de soldadura de la interface, independientemente de la geometría de la herramienta y de los espesores a soldar. En la Fig. 4a se muestra la evolución de IH en función

TABLA 3. Análisis dimensional de los puntos de soldadura

\begin{tabular}{lccccc}
\hline Probeta & $\begin{array}{c}\mathbf{P} \\
(\mathbf{m m})\end{array}$ & $\begin{array}{c}\mathbf{K r} \\
(\mathbf{m m})\end{array}$ & $\begin{array}{c}\mathbf{A} \\
(\mathbf{m m})\end{array}$ & $\begin{array}{c}\mathbf{I H} \\
\mathbf{( m m})\end{array}$ & $\mathbf{H}$ \\
\hline $1-680$ & 1,69 & 1,07 & 1,9 & 1,15 & 0,05 \\
$2-680$ & 1,6 & 1,05 & 2 & 1,05 & 0,1 \\
$3-680$ & 1,53 & 1,02 & 1,77 & 0,98 & 0,17 \\
$4-680$ & 1,41 & 0,65 & 3,3 & 0,81 & 0,29 \\
$5-680$ & 1,31 & 0,55 & 3,95 & 0,76 & 0,39 \\
$6-680$ & 1,14 & 0,62 & 4,3 & 0,68 & 0,56 \\
$1-903$ & 1,69 & 1,09 & 1,8 & 1,2 & 0,05 \\
$3-903$ & 1,54 & 0,96 & 1,95 & 0,97 & 0,16 \\
$4-903$ & 1,42 & 0,66 & 3,2 & 0,8 & 0,28 \\
$5-903$ & 1,33 & 0,78 & 4,06 & 0,89 & 0,37 \\
$5-903$ & 1,25 & 0,51 & 4,91 & 0,58 & 0,45 \\
$6-903$ & 1,18 & 0,5 & 4,8 & 0,65 & 0,52 \\
$1-1200$ & 1,69 & 0,5 & 0,61 & 1,12 & 0,05 \\
$2-1200$ & 1,6 & 0,38 & 1,1 & 0,98 & 0,1 \\
$3-1200$ & 1,5 & 0,38 & 1,3 & 0,95 & 0,2 \\
$3-1200$ & 1,56 & 0,4 & 1,2 & 1,03 & 0,14 \\
$3-1200$ & 1,5 & 0,5 & 1,5 & 0,98 & 0,2 \\
$4-1200$ & 1,4 & 0,5 & 1,96 & 1,03 & 0,3 \\
$5-1200$ & 1,3 & 0,44 & 2,3 & 0,8 & 0,4 \\
$6-1200$ & 1,2 & 0,3 & 2,6 & 0,6 & 0,5 \\
\hline & & & & &
\end{tabular}

de la indentación $H$, para todas las velocidades de rotación estudiadas. Se aprecia como IH disminuye con $\mathrm{H}$, como se observó previamente en las Fig. 3 (a-1) para las tres velocidades de rotación estudiadas. Es de hacer notar que para un mismo nivel de indentación, IH no presenta grandes variaciones con la velocidad de rotación empleada.

En la Fig. 4b se aprecia la evolución de la longitud efectiva de la junta A en función de la indentación. Puede verse como A aumenta con la indentación poniendo de manifiesto el efecto que tiene el hombro de la herramienta sobre el flujo plástico de material en la zona de la interface. El hombro de la herramienta de soldadura es el máximo responsable por el aporte térmico (Mishra y Mahoney, 2007; Lohwasser y Chen, 2010). De esta manera, uniones soldadas con altos valores de indentación presentan elevados valores de la longitud efectiva de junta A, como se aprecia en la Fig. 4b. Del análisis de la Fig. 4b surge también que para una dada indentación, el aumento de la velocidad de rotación de la herramienta tiene como consecuencia la disminución de la sección efectiva de junta. Este efecto se vuelve más notorio al soldar con 1200 RPM. Los resultados obtenidos son consistentes con lo que se reporta en la bibliografía (Yang et al., 2010; Yuan et al., 2011; Piccini y Svoboda, 2015a ; Rao et al., 2015).

El calor aportado por la herramienta para bajas velocidades de rotación es suficiente para plastificar y deformar al material alrededor del pin, manteniendo altos niveles de viscosidad en regiones cercanas al mismo. Este efecto resulta en la ruptura de la interface entre ambas chapas, mezclando ambos materiales y produciendo la unión soldada con altos valores de longitud efectiva de unión (Rao et al., 2015). Por el contrario, elevar la velocidad de rotación produce que la interface se desplace hacia arriba y se acerque a la pared lateral del pin. En la Fig. 4c se aprecia como $\mathrm{Kr}$ disminuye con la indentación y con la velocidad de rotación de la herramienta. Un correcto balance entre $\mathrm{A}, \mathrm{IH}$ y $\mathrm{Kr}$ será el que maximice las propiedades mecánicas de las uniones soldadas (Piccini y Svoboda, 2015a).

\subsection{Análisis microestructural}

A fin de lograr una mejor comprensión de los fenómenos que tienen lugar dentro de la unión soldada cuando se modifican los parámetros de soldadura, se analizó la microestructura en la zona agitada asociada a la interface entre ambas chapas. En particular, se centró el análisis sobre la agitación de ambos materiales, la formación del hook y la evolución de la junta hasta la pared lateral del key-hole (Fig. 5). A medida que la penetración aumenta, el flujo de material de cada chapa que es agitado entre sí aumenta. El hook queda definido por el último punto visible de interface entre ambas chapas. Este se encuentra 
Efecto de la velocidad de rotación y la indentación en soldadura de punto por fricción agitación • 9
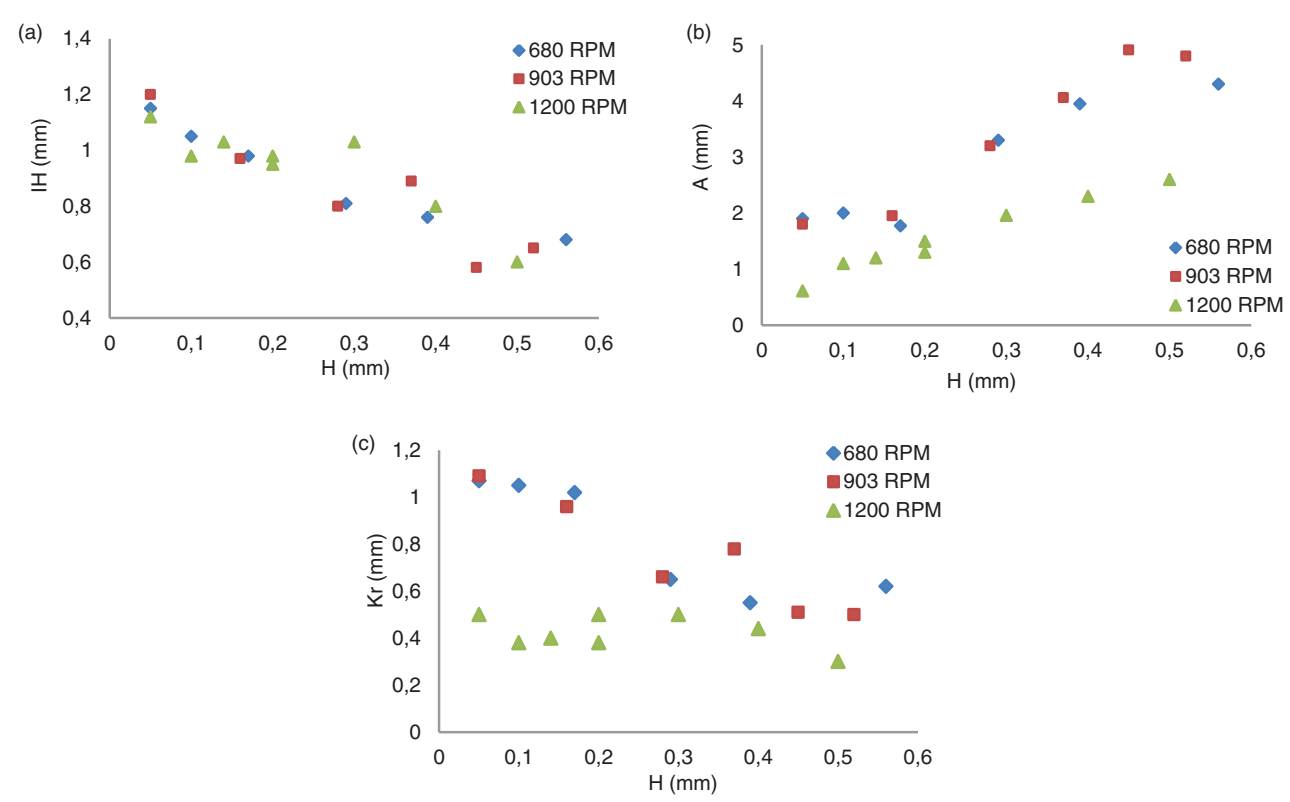

Figure 4. Evolución de: a) IH, b) A y c) Kr en función de la indentación.

(a)

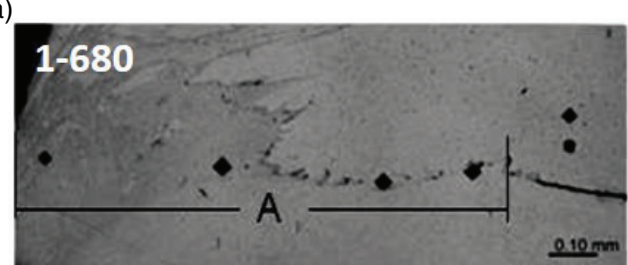

(c)

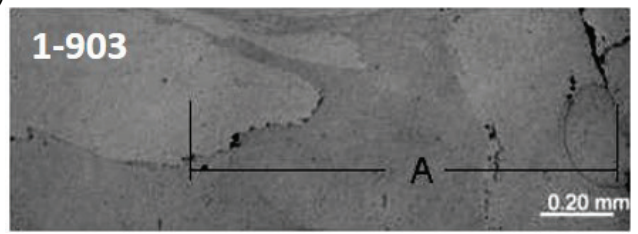

(e)

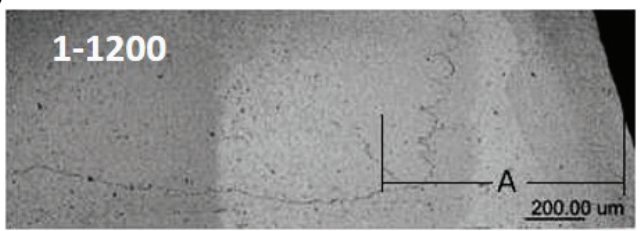

(b)

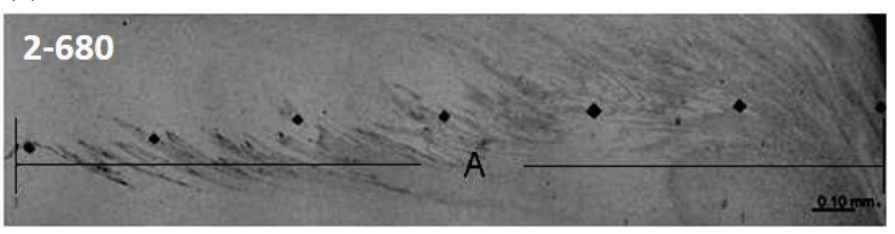

(d)

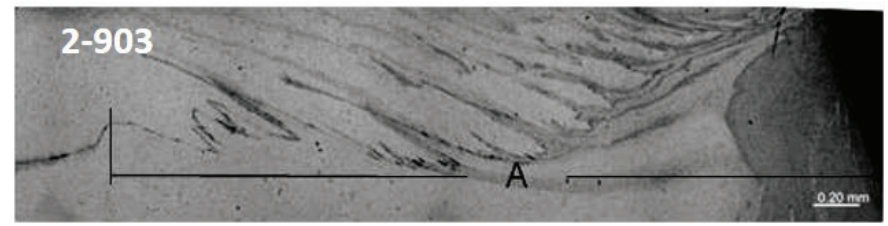

(f)

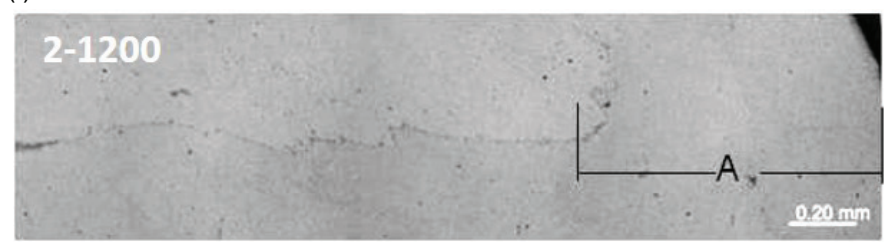

Figure 5. Micrografías de las interfaces.

dentro de la zona agitada y se convierte en un defecto geométrico que afecta a la calidad del punto de soldadura, ya que la fractura podrá iniciarse en dicho punto cuando la junta es solicitada por una carga externa (Tran et al., 2009). Puede verse en la Fig. 5 que en general el hook se ubica por arriba de la línea de la interface entre ambas chapas, donde comienza la agitación de ambos materiales, discontinuando la línea de óxido original de la interface. En la Fig. 5 (a-f) puede apreciarse la agitación y mezcla sufrida por los materiales en estado plástico. Se observa con mayor detalle como la longitud de unión efectiva (A) aumenta con la indentación (Fig. 5 (a-b)). Este efecto estaría relacionado con que la mayor proporción del aporte térmico se produce en la zona de contacto entre el hombro de la herramienta y la chapa superior (Hirasawa et al., 2010). Por lo tanto, cuanto más cerca esté el hombro de la interface, mayor será la agitación de los materiales y más eficiente la unión metalúrgica. 
En las Fig. 5 (a-f) se ve también como la longitud efectiva de unión disminuye con el aumento de la velocidad de rotación de la herramienta. Se ha reportado previamente que mayores longitudes de unión han sido obtenidas al reducir la velocidad de rotación de la herramienta, con un hook relativamente plano que tiende a desaparecer fuera de la zona agitada (Yang et al., 2010; Yuan et al., 2011; Rao et al., 2015). Al soldar con elevadas velocidades de rotación, el hook tiende a curvarse y desplazarse hacia arriba dentro de la chapa superior, lo cual reduce la longitud efectiva de unión (Yuan et al., 2011). Este efecto concuerda con lo que se aprecia en las Fig. 3 (a-l) y Fig. 5 (a-f). El aporte térmico aumenta con la velocidad de rotación reduciendo la viscosidad del material bajo el hombro y alrededor del pin. Así, mayores RPM producen un mayor flujo plástico, más material es agitado por unidad de tiempo y menores componentes de corte y forjado son necesarias por parte de la herramienta para deformar el material. Sin embargo, este efecto se concentra en las cercanías del pin reduciendo la SZ con el aumento de la velocidad de rotación, en concordancia con lo apreciado en la bibliografía (Rao et al., 2015). Por lo tanto, el aumento del aporte térmico (por medio del aumento de la velocidad de rotación de la herramienta) no produce la optimización de las uniones soldadas por FSSW de aleaciones de aluminio, dado que el flujo plástico que se produce tiende a curvar el hook, disminuyendo la sección resistente de las uniones.

\subsection{Microdureza vickers}

La aleación AA6063-T6 es termoenvejecible y luego de la soldadura las propiedades mecánicas evolucionan en el tiempo debido a un envejecimiento natural (Ferjutz y Davis, 1993; Jenney y O’Brien, 2001; Kou, 2003). En este sentido, a fin de realizar comparaciones, los ensayos de microdureza se realizaron luego de treinta días de haber realizado las uniones soldadas. La zona más relevante desde el punto de vista microestructural es la asociada al hook, dado que por allí se espera que se propague el frente de fractura interfacial. En el mismo sentido, se ha reportado previamente que existen mínimas diferencias en los valores de dureza asociados a las chapas superior e inferior de una junta solapada (Chowdhury et al., 2012). Finalmente, dada la relación de espesores de las chapas siendo soldadas, la fractura seria interfacial (propagándose desde el hook hasta la pared lateral del pin) o circunferencial (propagándose desde el hook hasta la superficie libre de la chapa superior). Por lo tanto, el perfil de
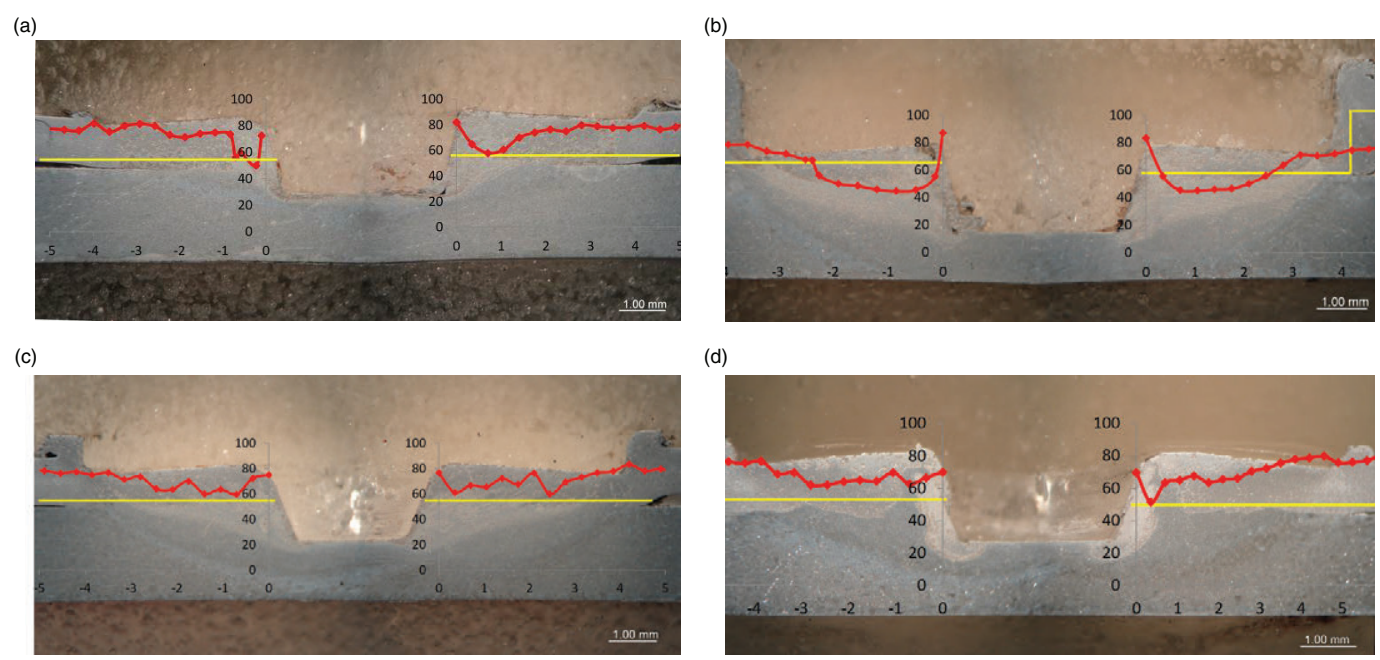

(d)

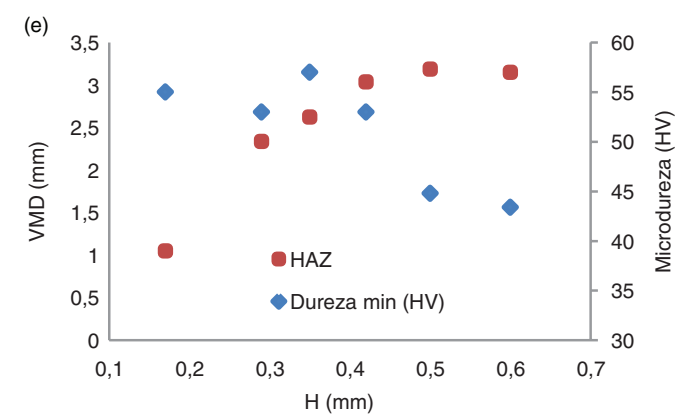

Figure 6. Perfiles de microdureza Vickers: a) 2-680, b) 5-680, c) 2-903, d) 2-1200 y e) Volumen de material degradado y dureza mínima en función de la indentación. 
microdureza se realizó sobre una línea a la altura del mismo (sobre la chapa de AA5052-H32), con mediciones cada $350 \mu \mathrm{m}$. Los resultados obtenidos se aprecian en las Fig. 6 (a-d). La línea amarilla indica la zona de medición.

Puede verse en dichas figuras como la dureza disminuye desde el material base $(70 \mathrm{HV})$ hasta que se alcanza el valor mínimo en la transición entre la zona afectada por el calor y la zona termomecánicamente afectada (TMAZ). Este valor mínimo de dureza alcanzó los $42 \mathrm{HV}$ para las máximas indentaciones (Fig. 6b). Este comportamiento está relacionado con el efecto que el ciclo termomecánico de la soldadura tiene sobre el material base (el cual se encontraba en una condición H32), produciendo algún grado de recristalización (Ferjutz y Davis, 1993; Jenney y O’Brien, 2001; Mishra y Mahoney, 2007; Chowdhury et al., 2012; Jeon et al., 2012).

En las cercanías del perfil del pin (SZ) la estructura presenta una estructura de granos refinados y equiaxiales lo que produce un aumento de la dureza, alcanzando los $85 \mathrm{HV}$. Asimismo, debe considerarse que en dicha zona puede presentarse una mezcla con el material de la chapa inferior (AA6063-T6), de mayor dureza, contribuyendo al aumento de dureza registrado (Jeon et al., 2012).

Al variar la velocidad de rotación de la herramienta, no se producen cambios significativos en los perfiles de microdureza. Sin embargo, puede observase una diferencia en estos perfiles a medida que aumenta la indentación. No solo la dureza mínima disminuye al aumentar la indentación sino que también aumenta el volumen de material degradado (VMD) por el ciclo termomecánico de la soldadura (Fig. 6e), definido por la distancia horizontal entre el punto donde se alcanza la dureza del material base y la pared lateral del pin. Este efecto estaría asociado al mayor ciclo térmico sufrido en la zona analizada, al aumentar la indentación de la herramienta. Esta tendencia se observó para las tres velocidades de rotación estudiadas.

\subsection{Peel test}

En la Fig. 7 se aprecia la evolución de la carga de rotura en el ensayo de Peel Test con la indentación de la herramienta, para las diferentes velocidades de rotación analizadas. Se puede observar como la carga de rotura aumenta, hasta alcanzar un máximo, y luego disminuye con la penetración de la herramienta. Estos resultados concuerdan con lo que se ha reportado previamente para diferentes configuraciones de juntas (Yuan et al., 2011; Piccini y Svoboda, 2015a). En la Fig. 7, los puntos indicados con una " $x$ " representan aquellas uniones soldadas que fallaron con un modo de fractura tipo interfacial. La superficie de fractura interfacial puede apreciarse en la Fig. 8a. Este modo de fractura tiene asociadas elevadas cargas de rotura, alcanzando los $5800 \mathrm{~N}$ para $903 \mathrm{RPM}$. Los puntos indicados con un "+", corresponden a las probetas que fallaron

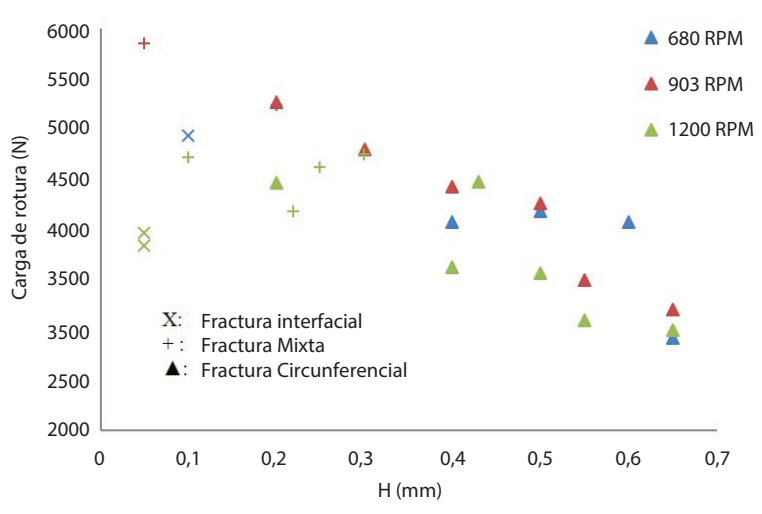

Figure 7. Evolución de la carga de rotura en Peel Test con la indentación. (a)
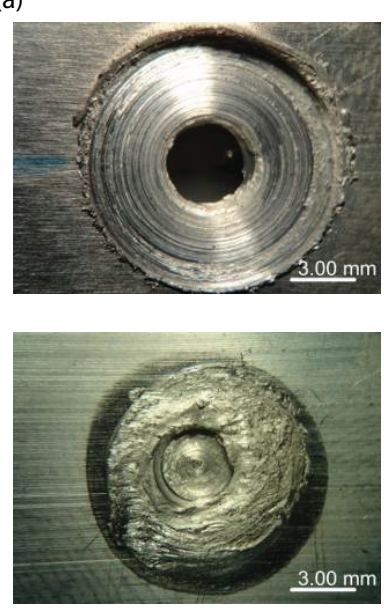

(b)
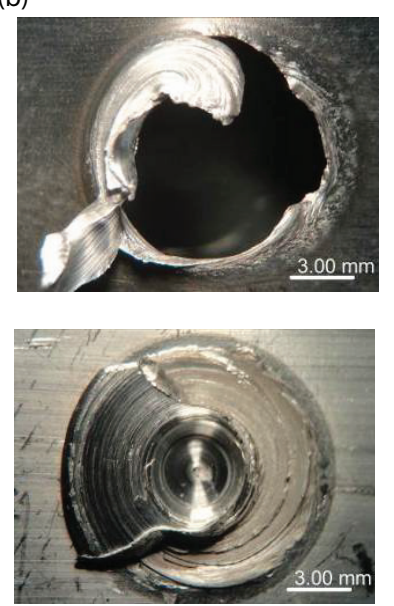

(c)
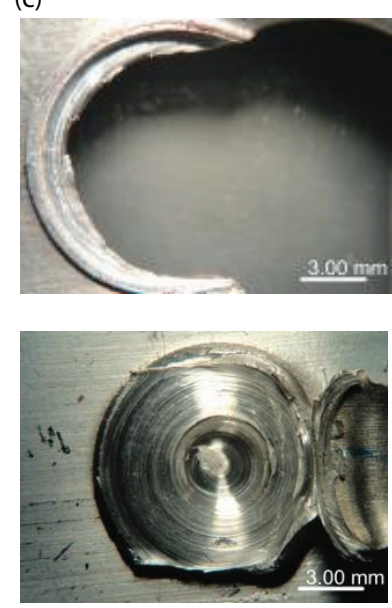

FIGURE 8. Superficies de fractura: a) 1-680, b) 2-680 y c) 3-1200. 
con una fractura tipo mixta (Fig. 8b), mientras que los demás puntos presentaron fracturas circunferenciales (Fig. 8c). Si bien mayores indentaciones implican mayor aporte térmico sobre la interface y el aumento de la dimensión A (Piccini y Svoboda, 2015a), la disminución excesiva del espesor remanente de la chapa superior (IH) produce la disminución de la carga de rotura (Piccini y Svoboda, 2015a; Rao et al., 2015), siempre adoptando un modo de fractura circunferencial. La fractura tipo mixta fue solo apreciada al soldar con 1200 RPM. Este modo de fractura se encuentra directamente relacionado con el flujo de material que se produce al soldar con elevadas velocidades de rotación. Como fue previamente comentado, al soldar con 1200 RPM la morfología del hook cambia, curvándose hacia arriba (reducción excesiva de $\mathrm{Kr}$ ) y hacia zonas cercanas al key-hole, produciendo este tipo de modo de fractura.

Uniones soldadas con niveles de indentación excesivamente bajos $(H<0,05)$ no pudieron ser realizadas, por lo que $\mathrm{H}=0,05$ representa el límite de soldabilidad de la configuración estudiada, con la herramienta utilizada. Al soldar con $\mathrm{H}$ alrededor de 0,1-0,2 $\mathrm{mm}$ las cargas de rotura fueron máximas. Cuando la indentación supera dicho valor, las cargas de rotura disminuyeron conforme a la reducción excesiva de IH y $\mathrm{Kr}$.

En la Fig. 7 puede también verse como al soldar con 1200 RPM la carga de rotura es menor que cuando se suelda con menores velocidades de rotación de la herramienta, para todos los niveles de indentación estudiados. Como se mencionó anteriormente, la modificación del flujo de material afecta tanto a la posición final del hook como al tamaño de la zona agitada. De esta manera, para valores bajos de indentación, donde la longitud efectiva de junta $\mathrm{A}$ es quien controla la resistencia de la unión (Piccini y Svoboda, 2015b), se aprecia una gran diferencia en la carga de rotura al soldar con diferentes velocidades de rotación. Al incrementar la indentación, $\mathrm{IH}$ y $\mathrm{Kr}$ son quienes controlan la capacidad portante de la unión (Piccini y Svoboda, 2015 b), por lo que el efecto de la velocidad de rotación sobre la carga de rotura de las uniones es menor. Estos resultados son coherentes con lo apreciado en las Figs. 4 y 5.

Un correcto balance entre las tres dimensiones características maximizará las propiedades mecánicas de las uniones soldadas (Zhang et al., 2011; Piccini y Svoboda, 2015b; Rao et al., 2015).

Los valores obtenidos de carga de rotura han sido mejorados respecto a lo previamente reportado, teniendo en cuenta los espesores y materiales que se sueldan en el presente trabajo, y los parámetros de soldadura empleados (Hirasawa et al., 2010; Jeon et al., 2012; Bozurt y Bilici, 2013; Cox et al., 2014; Piccini y Svoboda, 2015b). No solo se han optimizado las cargas de rotura en PT, sino que, además, dichas cargas han sido obtenidas en una configuración de junta que presenta un $40 \%$ de disminución en peso a lo previamente reportado (Piccini y Svoboda, 2015b). Finalmente, es de hacer notar que las propiedades mecánicas obtenidas son incluso superiores a las obtenidas mediante RSW y con menor consumo energético (Pereira et al., 2010), lo cual pone de manifiesto el potencial tecnológico que este proceso presenta.

\section{CONCLUSIONES}

- Se realizaron puntos de soldadura en aleaciones de aluminio disimiles del tipo AA5052-AA6063, de 1 y $2 \mathrm{~mm}$ de espesor respectivamente, mediante FSSW. Se analizó el efecto de la indentación y de la velocidad de rotación de la herramienta, obteniéndose uniones soldadas sin defectos y con excelente aspecto superficial.

- La calidad superficial de los puntos soldados mejora con el aumento de la velocidad de rotación. Por otro lado, al elevar la velocidad de rotación, la viscosidad en las cercanías de la herramienta disminuye y por lo tanto la capacidad de mezcla del pin también lo hace. Esto produce un hook curvado hacia arriba dentro de la chapa superior, disminuyendo la sección efectiva de junta para 1200 RPM.

- Los perfiles de dureza reflejan una disminución de la misma desde el material base hasta la transición entre la HAZ y la TMAZ. Luego, la dureza aumenta dentro de la SZ debido al refinamiento de grano que se produce durante la soldadura. Finalmente, se observó que el tamaño del volumen de material degradado por el ciclo termomecánico de la soldadura aumenta con la indentación de la herramienta.

- A medida que aumenta la indentación de la herramienta aumenta la longitud efectiva de junta A, a expensas de la disminución del espesor remanente IH. El aumento de la velocidad de rotación de la herramienta produce cambios en la morfología del hook disminuyendo la dimensión Kr. Esto produce una disminución de la sección resistente de los puntos de soldadura realizados con 1200 RPM. La optimización de dichas longitudes, maximiza las propiedades mecánicas, por lo cual la utilización de 1200 RPM no es adecuado.

- Se observó como a medida que aumenta la indentación, aumenta la carga de rotura en el ensayo PT, alcanzando un valor máximo de $5800 \mathrm{~N}$ para $0,1 \mathrm{~mm}$ de indentación. Este valor se obtuvo para 903 RPM. Sin embargo, esta tendencia tiene un límite, ya que para valores superiores de $\mathrm{H}$ la carga de rotura disminuye debido a la reducción excesiva de $\mathrm{IH}$.

- Al soldar con bajas velocidades de rotación, valores de indentación bajos y medios presentan 
fracturas del tipo interfacial y circunferencial respectivamente. Las máximas cargas de rotura presentaron fracturas del tipo interfacial y circunferencial poniendo de manifiesto como el balance entre A e IH controla el comportamiento durante los ensayos de PT. Puntos de soldadura con indentaciones elevadas presentaron fracturas circunferenciales con cargas de rotura bajas, como consecuencia de una elevada reducción de IH. Al soldar con 1200 RPM un modo de fractura tipo mixto fue apreciado para niveles medios de indentación. Este modo de fractura no presenta cargas de rotura máximas, como consecuencia de la morfología que presenta el hook.

- Las propiedades mecánicas obtenidas en los ensayos de PT son mayores a lo reportado en la bibliografía, ya sea al soldar mediante FSSW o mediante RSW. Esto pone de manifiesto la optimización del procedimiento de soldadura y el potencial tecnológico que presenta.

\section{AGRADECIMIENTOS}

Los autores desean agradecer a PSA PEUGEOT CITROEN ARGENTINA y a la Universidad de Buenos Aires por el financiamiento para el desarrollo del presente trabajo.

\section{REFERENCIAS}

ASTM E384 (2009). Standard Test Method for Microindentation Hardness of Materials, ASTM, USA.

Bozkurt, Y., Bilici, M. (2013). Application of Taguchi approach to optimize of FSSW parameters on joint properties of dissimilar AA2024-T3 and AA5754-H22 aluminum alloys. Mater. Design 51, 513-521. http://dx.doi.org/10.1016/j. matdes.2013.04.074

Chowdhury, S., Chen, D., Bhole, S., Cao, X., Wanjara, P. (2012). Lap shear strength and fatigue life of friction stir spot welded AZ31 magnesium and 5754 aluminum alloys. Mater. Sci. Eng. A. 556, 500-509. http://dx.doi. org/10.1016/j.msea.2012.07.019.

Cox, C., Gibson, B., Strauss, A., Cook, G. (2014). Energy input during friction stir spot welding. J. Manuf. Process. 16 (4), 479-484. http://dx.doi.org/10.1016/j.jmapro.2014.05.007.

European Aluminium Association (2015). The Aluminium Automotive Manual, http://c.ymcdn.com/sites/www.aec. org/resource/resmgr/PDFs/1-Intro_2015.pdf [accessed 26. 06.15]

Ferjutz, K., Davis, J.R. (1993). Metal Handbook. Welding Brazing and Soldering. Vol. 6, ASM International, USA.

Francesco, L., Svoboda, H. (2013). Efecto de las variables de proceso de Soldadura de Punto por Fricción Agitación (FSSW) de aluminio en las propiedades mecánicas. FIUBA, Buenos Aires, Argentina.

Hirasawa, S., Badarinarayan, H., Okamoto, K., Tomimura, T., Kawanami, T. (2010). Analysis of effect of tool geometry on plastic flow during friction stir spot welding using particle method. J. Mater. Process. Technol. 210 (11), 14551463. http://dx.doi.org/10.1016/j.jmatprotec.2010.04.003.
Jenney, C.L., O'Brien, L. (2001). Welding Handbook. Welding Science and Technology, Vol. 1, ASM International, USA.

Jeon, C., Hong, S., Kwon, Y., Cho, H., Han, H. (2012). Material properties of friction stir spot welded joints of dissimilar aluminum alloys. Trans. Nonferrous Met. Soc. China. 22 (3), s605-s613. http://dx.doi.org/10.1016/ S1003-6326(12)61772-5.

Kou, S. (2003). Welding Metallurgy. Second Edition, John Wiley \& Sons, Hoboken, Canadá

Lathabai, S., Painter, M., Cantin, G., Tyagi, V. (2006). Friction spot joining of an extruded Al-Mg-Si alloy. Scripta Mater. 55 (10), 899-902. http://dx.doi.org/10.1016/j.scriptamat. 2006.07.046.

Lohwasser, D., Chen, Z. (2010). Friction Stir Welding. From basics to applications. Woodhead Publishing Limited, Oxford Cambridge.

Mishra, R., Mahoney, M. (2007). Friction Stir Welding and Processing. ASM International, Materials Park, Ohio.

Pereira, A., Ferreira, J., Loureiro, A., Costa, J., Bártolo, P. (2010). Effect of process parameters on the strength of resistance spot welds in 6082-T6 aluminium alloy. Mater. Design 31 (5), 2454-2463. http://dx.doi.org/10.1016/j. matdes.2009.11.052.

Piccini, J., Svoboda, H. (2015a). Effect of the tool penetration depth in Friction Stir Spot Welding (FSSW) of dissimilar aluminum alloys. Proc. Mat. Sc. 8, 868-877. http://dx.doi. org/10.1016/j.mspro.2015.04.147.

Piccini, J., Svoboda, H. (2015b). Effect of pin length on Friction Stir Spot Welding (FSSW) of dissimilar AluminumSteel joints. Proc. Mat. Sc. 9, 504-513. http://dx.doi. org/10.1016/j.mspro.2015.05.023.

Rao, H., Yuan, W., Badarinarayan, H. (2015). Effect of process parameters on mechanical properties of friction stir spot welded magnesium to aluminum alloys. Mater. Design 66 (Part A), 235-245. http://dx.doi.org/10.1016/j. matdes.2014.10.065.

Shome, M., Tumuluru, M. (2015). Welding and Joining of Advanced High Strength Steels (AHSS). Elsevier, Cambridge, UK.

Tozaki, Y., Uematsu, Y., Tokaji, K. (2007). Effect of tool geometry on microstructure and static strength in friction stir spot welded aluminum alloys. Int. J. Mach. Tool. Мапи. 47 (15), 2230-2236. http://dx.doi.org/10.1016/j. ijmachtools.2007.07.005.

Tran, V., Pan, J., Pan, T. (2009). Effects of processing time on strengths and failure modes of dissimilar spot friction welds between aluminum 5754-O and 7075-T6 sheets. $J$. Mater. Process. Technol. 209 (8), 3724-3739. http://dx.doi. org/10.1016/j.jmatprotec.2008.08.028.

Vander Voort, G.F. (2004). Metal Handbook. Metallography and Microstructures, Vol. 9, ASM International, USA

Wang, D.-A., Lee, S.-C. (2007). Microstructures and failure mechanisms of frictions stir spot welds of aluminum 6061-T6 sheets. J. Mater. Process. Technol. 186 (1-3), 291-297. http://dx.doi.org/10.1016/j.jmatprotec. 2006.12.045.

Yang, Q. Mironov, S. Sato, Y, Okamoto, K. (2010). Material flow during friction stir spot welding. Mat. Sci. Eng. A 527 (16-17), 4389-4398. http://dx.doi.org/10.1016/j. msea.2010.03.082.

Yuan, W., Mishra, R., Webb, S., Chen, Y., Carlson, B., Herling, D., Grant, G. (2011). Effect of tool design and process parameters on properties of $\mathrm{Al}$ alloy 6016 friction stir spot welds. J. Mater. Process. Technol. 211 (6), 972-977. http:// dx.doi.org/10.1016/j.jmatprotec.2010.12.014.

Zhang, Z., Yang, X., Zhang, J., Zhou, G., Xu, X., Zou, B. (2011). Effect of welding parameters on microstructure and mechanical properties of friction stir spot welded 5052 aluminum alloy. Mater. Design 32, 4461-4470. http:// dx.doi.org/10.1016/j.matdes.2011.03.058. 